\title{
Influence of the Phase on the Entanglement between a Jaynes-Cummings Atom and an Isolated Atom
}

\author{
C.-Q. PANG AND M.-L. LIANG* \\ Physics Department, School of Science, Tianjin University, Tianjin 300072, China
}

(Received June 4, 2012; in final form March 26, 2014)

\begin{abstract}
A scheme is presented to generate an entangled atomic state, for which the quantum phase affects the atomatom entanglement when the atom interacts with external light fields. The quantum phase enhances the entanglement and extends the time that the system stays in stronger entanglement state or the phase makes the system more robust. It is also shown that the quantum phase is able to delay the entanglement sudden death and hasten the entanglement revival.
\end{abstract}

DOI: 10.12693/APhysPolA.125.1069

PACS: 03.65.Ud, 42.50.Dv, 03.67.-a

\section{Introduction}

In classical information, the elementary unit is the bit (or cbit for classical bit), which is a classical system with only two states 0 and 1 . If there are many cbits, the state of the whole system is the direct product of the state of each cbit. Being an extension of the classical information theory, the quantum information theory has attracted more and more attention [1-3]. In quantum information, the analog of the classical bit is the qubit or quantum bit. It is a two-dimensional quantum system like a spin- $1 / 2$, a photon polarization, an atom with two relevant states, etc. Different from cbits, qubits can be in entangled states [1-4]. It is believed that entanglement is the main resource in quantum information processing such as quantum communication and quantum computation. However, entanglement in multipartite quantum systems may deteriorate due to interaction with the environment. It is really an obstacle for realizing quantum information processing. Besides the application to quantum information processing realizations, a deeper understanding of disentanglement is also significant to quantum fundamentals. Therefore, the loss of entanglement is an important subject to research.

Recently, it has been shown that entanglement may terminate abruptly in a finite time [5-7]. This phenomenon is currently termed as entanglement sudden death (ESD). Further, if a system interacts with a thermal $[8,9]$ or squeezed [10] reservoirs, the entanglement is lost in a finite time, too. Besides ESD, other nonclassical correlations as described by violations of classical inequalities have also the property of finite decays (for dissipative systems) and analogous periodic vanishings (for unitary systems) [11], which means these sudden vanishings are universal phenomena and represent a rather general nature. While studying coupled nonlinear oscillators, it is pointed out that entanglements of differ-

* corresponding author; e-mail: mailinliang@tju.edu.cn; mailinliang@163.com ent subsystems are not independent [12]. Moreover, in [13] it is shown that an external driving field coupling to one of the nonlinear oscillator mode can be responsible for entanglement return to the system considered. These results tell us that it is meaningful to investigate the entanglements of different subsystems in a system.

Two-level atom is one of the promising candidates for the qubit [14]. Light field can be used to change the states of the atom [15]. Interaction of the light field with a two-level atom is governed by the Jaynes-Cummings (JC) model [16]. For a quantum system, two-qubit entanglement is the fundamental one and is believed to be essential to implement quantum processes such as quantum computation $[2,3,14]$. So the study of the system of two atoms plus light is basically necessary.

In previous work, ESD based on the JC model [16] has been intensively studied and acquired significant achievements [17-28]. It is demonstrated that forms of initial wave functions, the photon number in the cavity, non-resonant interaction have direct effects on the appearance of ESD. Though great progress has been made, effects of quantum phase on ESD are seldom addressed, which is partly due to the fact that most of the former studies use $X$-type atomic density matrices and the quantum phase does not affect the entanglement in such kinds of states.

Here in this article, we present a scheme to generate an entangled atomic state that the quantum phase does not affect the original atom-atom entanglement, but will affect the atom-atom entanglement when there is an environment, the light field. To quantify the atom-atom and atom-field entanglement in a unified way, we use negativity $[29,30]$ to measure the entanglement, which provides the necessary and sufficient condition for the existence of entanglement in a qubit-qubit or qubit-qutrit system. In some cases, the concurrence [31-33] will be given directly. The scheme to generate the initial atomic state is given in the next section. The main calculations about the entanglement are presented in the third section. The final section is a summary. 


\section{The initial state}

In most of previous studies [17-25], the atom-atom density matrix is the $X$-type one

$$
\rho_{A 1 A 2}=\left[\begin{array}{cccc}
\rho_{11} & 0 & 0 & \rho_{14} \\
0 & \rho_{22} & \rho_{23} & 0 \\
0 & \rho_{32} & \rho_{33} & 0 \\
\rho_{41} & 0 & 0 & \rho_{44}
\end{array}\right] \text {. }
$$

Using the negativity measure of entanglement [29, 30], one can clearly see that the phases of the matrix elements in (1) do not affect the entanglement. According to the definition, the negativity is defined as multiplying the factor -2 by the sum of the negative eigenvalues of the partial transpose of the density matrix. For the density matrix (1), the partial transpose has the form

$$
\rho_{A 1 A 2}^{(\mathrm{PT})}=\left[\begin{array}{cccc}
\rho_{11} & 0 & 0 & \rho_{23} \\
0 & \rho_{22} & \rho_{14} & 0 \\
0 & \rho_{41} & \rho_{33} & 0 \\
\rho_{32} & 0 & 0 & \rho_{44}
\end{array}\right] .
$$

Its possible negative eigenvalues are

$$
\begin{aligned}
& \lambda_{1 n}=-\left[\sqrt{\left(\rho_{22}-\rho_{33}\right)^{2}+4\left|\rho_{14}\right|^{2}}-\left(\rho_{22}+\rho_{33}\right)\right] / 2, \\
& \lambda_{2 n}=-\left[\sqrt{\left(\rho_{11}-\rho_{44}\right)^{2}+4\left|\rho_{23}\right|^{2}}-\left(\rho_{11}+\rho_{44}\right)\right] / 2 .
\end{aligned}
$$

The negativity is calculated as

$$
E_{A 1 A 2}=\max \left(0,-2 \lambda_{1 n}\right)+\max \left(0,-2 \lambda_{2 n}\right) .
$$

Through the form of $(3)$, one sees that the entanglement measure (4) is independent of the phases of the matrix elements. It is also known that the atom-field interaction does not change the form of the $X$-type matrix form. So, to see the effects of quantum phases, we should have a non- $X$ type density matrix.

Here in this article we consider the case that there are two two-level atoms labeled $A 1$ and $A 2$ and they initially stay in the following entangled state (the condition is $q \neq 0)$ :

$$
\begin{aligned}
& \left|\psi_{A 1 A 2}(0)\right\rangle=\sqrt{q}\left(\cos \alpha\left|g_{1} g_{2}\right\rangle+\mathrm{e}^{\mathrm{i} \varphi} \sin \alpha\left|e_{1} e_{2}\right\rangle\right) \\
& \quad+\mathrm{e}^{\mathrm{i} \theta} \sqrt{1-q}\left|g_{1} e_{2}\right\rangle
\end{aligned}
$$

where $q, \alpha, \varphi, \theta$ are all real parameters, $\left|g_{j}\right\rangle,\left|e_{j}\right\rangle(j=1,2)$ are the ground and excited states of the two-level atoms. At first we provide a scheme to generate this state.

Various methods can be used to generate entangled atomic states, such as the atom-field interaction [34], the classical field transition [35], quantum measurement, etc. [24]. In our scheme, the first step is atom $A 1$ in a general state $c\left|e_{1}\right\rangle+d\left|g_{1}\right\rangle$ going through a cavity field in vacuum state $|0\rangle$. Using the Jaynes-Cummings model [16], at time $t_{1}$ the wave function for the atom-field system becomes

$$
\begin{aligned}
& \left|\psi_{A 1 f}\left(t_{1}\right)\right\rangle=\mathrm{e}^{\mathrm{i} \Delta / 2 t_{1}} d\left|g_{1}\right\rangle|0\rangle \\
& +c\left[\left(\cos A t_{1}-\mathrm{i} \frac{\Delta}{2 A} \sin A t_{1}\right)\left|e_{1}\right\rangle|0\rangle\right. \\
& \left.-\mathrm{i} \frac{\lambda_{1}}{A} \sin A t_{1}\left|g_{1}\right\rangle|1\rangle\right],
\end{aligned}
$$

where $\lambda_{1}$ is the atom-field coupling constant, $\Delta$ - the detuning between light frequency and the atomic transition frequency and the factor $A$ is $A=\lambda_{1} \sqrt{1+\Delta^{2} /\left(4 \lambda_{1}^{2}\right)}$. In deriving (6), the evolution results have been used

$$
\begin{aligned}
& U\left(t_{1}\right)\left|e_{1}\right\rangle|n\rangle \\
& \quad=a_{e e}\left(n, t_{1}\right)\left|e_{1}\right\rangle|n\rangle+a_{e g}\left(n, t_{1}\right)\left|g_{1}\right\rangle|n+1\rangle, \\
& U\left(t_{1}\right)\left|g_{1}\right\rangle|n\rangle \\
& \quad=a_{g g}\left(n, t_{1}\right)\left|g_{1}\right\rangle|n\rangle+a_{g e}\left(n, t_{1}\right)\left|e_{1}\right\rangle|n-1\rangle,
\end{aligned}
$$

where $U\left(t_{1}\right)$ is the evolution operator for the JC model $[16,18-20], n$ is an integer representing the photon number in the state. The time-dependent coefficients in $(7),(8)$ are

$$
\begin{aligned}
& a_{e e}\left(n, t_{1}\right)=\cos \sqrt{n+1+\Delta^{2} /\left(4 \lambda_{1}^{2}\right)} T_{1} \\
& -\mathrm{i} \frac{\Delta \sin \sqrt{n+1+\Delta^{2} /\left(4 \lambda_{1}^{2}\right)} T_{1}}{2 \lambda_{1} \sqrt{n+1+\Delta^{2} /\left(4 \lambda_{1}^{2}\right)}}, \\
& a_{e g}\left(n, t_{1}\right)=-\mathrm{i} \frac{\sqrt{n+1} \sin \sqrt{n+1+\Delta^{2} /\left(4 \lambda_{1}^{2}\right)} T_{1}}{\sqrt{n+1+\Delta^{2} /\left(4 \lambda_{1}^{2}\right)}}, \\
& a_{g g}\left(n, t_{1}\right)=\cos \sqrt{n+\Delta^{2} /\left(4 \lambda_{1}^{2}\right)} T_{1} \\
& -\mathrm{i} \frac{\Delta \sin \sqrt{n+\Delta^{2} /\left(4 \lambda_{1}^{2}\right)} T_{1}}{2 \lambda_{1} \sqrt{n+\Delta^{2} /\left(4 \lambda_{1}^{2}\right)}}, \\
& a_{g e}\left(n, t_{1}\right)=-\mathrm{i} \frac{\sqrt{n} \sin \sqrt{n+\Delta^{2} /\left(4 \lambda_{1}^{2}\right)}}{\sqrt{n+\Delta^{2} /\left(4 \lambda_{1}^{2}\right)}} .
\end{aligned}
$$

Here a scaled time $T_{1}=\lambda_{1} t_{1}$ is defined. After atom $A 1$ exits the field, atom $A 2$ in ground state enters the field. Assuming the interaction time is $t_{2}$, the wave function (6) further changes into

$$
\begin{aligned}
& \left|\psi_{A 1 A 2 f}\left(t_{1}, t_{2}\right)\right\rangle=|0\rangle \mathrm{e}^{\mathrm{i} \Delta / 2 t_{1}+\mathrm{i} \Delta t_{2} / 2} d\left|g_{1} g_{2}\right\rangle \\
& +|0\rangle c\left[\mathrm{e}^{\mathrm{i} \Delta t_{2} / 2}\left(\cos A t_{1}-\mathrm{i} \frac{\Delta}{2 A} \sin A t_{1}\right)\left|e_{1} g_{2}\right\rangle\right. \\
& \left.\quad-\frac{\lambda_{1} \lambda_{2}}{A^{2}} \sin A t_{1} \sin A t_{2}\left|g_{1} e_{2}\right\rangle\right]-\mathrm{i} \frac{\lambda_{1}}{A} \sin A t_{1} \\
& \quad \times\left(\cos A t_{1}+\mathrm{i} \frac{\Delta}{2 A} \sin A t_{1}\right)\left|g_{1} g_{2}\right\rangle|1\rangle,
\end{aligned}
$$

which is a $W$-like state for atoms $A 1, A 2$ and the field. To make the atoms and field disentangled, a third atom $A 3$ in ground state is injected into the field. This atom interacts resonantly with the field. Choosing the interaction time so that $\lambda_{3} t_{3}=\pi / 2$, the state will undergo the changes

$$
|0\rangle\left|g_{3}\right\rangle \rightarrow|0\rangle\left|g_{3}\right\rangle, \quad|1\rangle\left|g_{3}\right\rangle \rightarrow-\mathrm{i}|0\rangle\left|e_{3}\right\rangle .
$$

The wave function (13) then becomes

$$
\begin{aligned}
& \left|\psi_{A 1 A 2 A 3 f}\left(t_{1}, t_{2}\right)\right\rangle=|0\rangle\left|g_{3}\right\rangle \mathrm{e}^{\mathrm{i} \Delta / 2 t_{1}+\mathrm{i} \Delta t_{2} / 2} d\left|g_{1} g_{2}\right\rangle \\
& +|0\rangle\left|g_{3}\right\rangle c\left[\mathrm{e}^{\mathrm{i} \Delta t_{2} / 2}\left(\cos A t_{1}-\mathrm{i} \frac{\Delta}{2 A} \sin A t_{1}\right)\left|e_{1} g_{2}\right\rangle\right. \\
& \left.-\frac{\lambda_{1} \lambda_{2}}{A^{2}} \sin A t_{1} \sin A t_{2}\left|g_{1} e_{2}\right\rangle\right]-|0\rangle\left|e_{3}\right\rangle \frac{\lambda_{1}}{A} \sin A t_{1}
\end{aligned}
$$




$$
\times\left(\cos A t_{1}+\mathrm{i} \frac{\Delta}{2 A} \sin A t_{1}\right)\left|g_{1} g_{2}\right\rangle .
$$

One finds that the field is disentangled from the atoms. After $A 3$ exits the field, its state is detected. If $A 3$ is detected to be in the ground state, atoms $A 1$ and $A 2$ will be in the entangled state

$$
\begin{aligned}
& \left|\psi_{A 1 A 2}\left(t_{1}, t_{2}\right)\right\rangle=\mathrm{e}^{\mathrm{i} \Delta / 2 t_{1}+\mathrm{i} \Delta t_{2} / 2} d\left|g_{1} g_{2}\right\rangle \\
& +c\left[\mathrm{e}^{\mathrm{i} \Delta t_{2} / 2}\left(\cos A t_{1}-\mathrm{i} \frac{\Delta}{2 A} \sin A t_{1}\right)\left|e_{1} g_{2}\right\rangle\right. \\
& \left.-\frac{\lambda_{1} \lambda_{2}}{A^{2}} \sin A t_{1} \sin A t_{2}\left|g_{1} e_{2}\right\rangle\right] .
\end{aligned}
$$

Using a classical microwave field [35], states of atom $A 2$ can have the transitions $\left|g_{2}\right\rangle \rightarrow\left|e_{2}\right\rangle,\left|e_{2}\right\rangle \rightarrow\left|g_{2}\right\rangle$. The wave function (16) becomes (5) with the coefficients written in new forms.

The concurrence for state (5) is $C_{A 1 A 2}=q|\sin (2 \alpha)|$. Numerical calculations show that the negativity is equal to the concurrence. Clearly, the phases $\varphi, \theta$ do not affect the entanglement. In the basis $\left|e_{1} e_{2}\right\rangle,\left|e_{1} g_{2}\right\rangle$, $\left|g_{1} e_{2}\right\rangle$, and $\left|g_{1} g_{2}\right\rangle$, the density matrix $\rho_{A 1 A 2}(0)=$ $\left|\psi_{A 1 A 2}(0)\right\rangle\left\langle\psi_{A 1 A 2}(0)\right|$ has the form

$$
\rho_{A 1 A 2}(0)=\left[\begin{array}{cccc}
\rho_{11} & 0 & \rho_{13} & \rho_{14} \\
0 & 0 & 0 & 0 \\
\rho_{31} & 0 & \rho_{33} & \rho_{34} \\
\rho_{41} & 0 & \rho_{43} & \rho_{44}
\end{array}\right],
$$

where

$$
\begin{aligned}
& \rho_{11}=q \sin ^{2} \alpha, \quad \rho_{33}=1-q, \quad \rho_{44}=q \cos ^{2} \alpha, \\
& \rho_{13}=\rho_{31}^{*}=\mathrm{e}^{\mathrm{i}(\varphi-\theta)} \sqrt{q(1-q)} \sin \alpha, \\
& \rho_{14}=\rho_{41}^{*}=\mathrm{e}^{\mathrm{i} \varphi} \sqrt{q} \sin \alpha \cos \alpha, \\
& \rho_{34}=\rho_{43}^{*}=\mathrm{e}^{\mathrm{i} \theta} \sqrt{q(1-q)} \sin \alpha .
\end{aligned}
$$

The matrix (17) is not the $X$-type one. When there is an external field, we expect new results about the quantum phases. In the next section, we mainly discuss the case that atom $A 2$ is exposed to the external field. Details about the case of $A 1$ interacting with the field will not be iterated. As we are mainly concerned with the effects of the phases, we choose the fixed values $q=0.5$ and $\alpha=\pi / 4$ in the numerical calculations when no specific statement is mentioned.

\section{Influence of phase on the entanglement}

We suppose that the field is initially in the zero or one photon state $|n\rangle(n=0,1)$. Atom $A 2$ interacts with this field and $A 1$ keeps isolated from this field. The initial state for $A 1, A 2$ and the cavity field is $\left|\psi_{A 1 A 2 F}(0)\right\rangle=$ $\left|\psi_{A 1 A 2}(0)\right\rangle|n\rangle$. At any time, the wave function becomes

$$
\begin{aligned}
& \left|\psi_{A 1 A 2 F}(t)\right\rangle=\sqrt{q} a_{g e}(n, t) \cos \alpha|n-1\rangle\left|g_{1} e_{2}\right\rangle \\
& \quad+\left(\sqrt{q} a_{g g}(n, t) \cos \alpha\left|g_{1} g_{2}\right\rangle\right. \\
& \quad+\sqrt{1-q} a_{e e}(n, t) \mathrm{e}^{\mathrm{i} \theta}\left|g_{1} g_{2}\right\rangle \\
& \left.\quad+\sqrt{q} a_{e e}(n, t) \mathrm{e}^{\mathrm{i} \varphi} \sin \alpha\left|g_{1} g_{2}\right\rangle\right)|n\rangle \\
& \quad+\left(\sqrt{q} a_{e g}(n, t) \mathrm{e}^{\mathrm{i} \varphi} \sin \alpha\left|e_{1} g_{2}\right\rangle\right.
\end{aligned}
$$

$$
\left.+\sqrt{1-q} a_{e g}(n, t) \mathrm{e}^{\mathrm{i} \theta}\left|g_{1} g_{2}\right\rangle\right)|n+1\rangle .
$$

For $n=0$, the function $a_{g e}(n, t)=0$ and the first term on the right side of (19) vanishes automatically. The density matrix for the whole system is $\rho_{A 1 A 2 F}(t)=$ $\left|\psi_{A 1 A 2 F}(t)\right\rangle\left\langle\psi_{A 1 A 2 F}(t)\right|$. Tracing over the field variables, the reduced density matrix for the two atoms is derived

$$
\rho_{A 1 A 2}(t)=\left[\begin{array}{cccc}
\rho_{11} & 0 & \rho_{13} & \rho_{14} \\
0 & \rho_{22} & 0 & \rho_{24} \\
\rho_{31} & 0 & \rho_{33} & \rho_{34} \\
\rho_{41} & \rho_{42} & \rho_{43} & \rho_{44}
\end{array}\right],
$$

where the nonzero matrix elements are

$$
\begin{aligned}
& \rho_{11}=q\left|a_{e e}(n, t) \sin \alpha\right|^{2}, \quad \rho_{22}=q\left|a_{e g}(n, t) \sin \alpha\right|^{2}, \\
& \rho_{33}=q\left|a_{g e}(n, t) \cos \alpha\right|^{2}+(1-q)\left|a_{e e}(n, t)\right|^{2}, \\
& \rho_{44}=q\left|a_{g g}(n, t) \cos \alpha\right|^{2}+(1-q)\left|a_{e g}(n, t)\right|^{2}, \\
& \rho_{13}=\rho_{31}^{*}=\sqrt{q(1-q)}\left|a_{e e}(n, t)\right|^{2} \mathrm{e}^{\mathrm{i}(\varphi-\theta)}, \\
& \rho_{14}=\rho_{41}^{*}=q a_{e e}\left(n, T_{1}\right) a_{g g}^{*}\left(n, T_{1}\right) \mathrm{e}^{\mathrm{i} \varphi} \cos \alpha \sin \alpha, \\
& \rho_{24}=\rho_{42}^{*}=\sqrt{q(1-q)}\left|a_{e g}(n, t)\right|^{2} \mathrm{e}^{\mathrm{i}(\varphi-\theta)} \sin \alpha, \\
& \rho_{34}=\rho_{43}^{*}=\sqrt{q(1-q)} a_{e e}\left(n, T_{1}\right) a_{g g}^{*}\left(n, T_{1}\right) \mathrm{e}^{\mathrm{i} \theta} \cos \alpha .
\end{aligned}
$$

Compared to the initial density matrix (17), three matrix elements $\rho_{22}, \rho_{24}$, and $\rho_{42}$ have appeared. We see that the atom-field interaction changes the form of the density matrix. In case of $q=1$, the density matrix (20) reduces to the $X$-type one. By some calculations, the equation for the eigenvalue $x$ of the partially transposed density matrix of (20) is found to be

$$
x^{4}-x^{3}+c x^{2}+d x+e=0,
$$

where the coefficients are

$$
\begin{aligned}
c= & \rho_{11} \rho_{44}+\rho_{22} \rho_{33}+\left(\rho_{11}+\rho_{44}\right)\left(\rho_{22}+\rho_{33}\right) \\
& -\left(\left|\rho_{13}\right|^{2}+\left|\rho_{14}\right|^{2}+\left|\rho_{24}\right|^{2}+\left|\rho_{34}\right|^{2}\right) \\
d= & \left(\rho_{11}+\rho_{22}\right)\left|\rho_{34}\right|^{2}+\left(\rho_{11}+\rho_{44}\right)\left|\rho_{14}\right|^{2} \\
& +\left(\rho_{11}+\rho_{33}\right)\left|\rho_{24}\right|^{2}+\left(\rho_{22}+\rho_{44}\right)\left|\rho_{13}\right|^{2} \\
& -\left(\rho_{14} \rho_{24} \rho_{34}+\rho_{41} \rho_{42} \rho_{43}\right)-\left(\rho_{11}+\rho_{44}\right) \rho_{22} \rho_{33} \\
& -\left(\rho_{22}+\rho_{33}\right) \rho_{11} \rho_{44}, \\
e & =\rho_{11} \rho_{44}\left(\rho_{22} \rho_{33}-\left|\rho_{14}\right|^{2}\right) \\
& +\rho_{11}\left(\rho_{14} \rho_{24} \rho_{34}+\rho_{41} \rho_{42} \rho_{43}\right)+\left|\rho_{13}\right|^{2}\left|\rho_{24}\right|^{2} \\
& -\rho_{11} \rho_{22}\left|\rho_{34}\right|^{2}-\rho_{11} \rho_{33}\left|\rho_{24}\right|^{2}-\rho_{22} \rho_{44}\left|\rho_{13}\right|^{2} .
\end{aligned}
$$

The phase $\theta$ disappears from the coefficients (23)-(25) and does not affect the entanglement evolution as the entanglement is determined by the eigenvalue $x$. The phase $\varphi$ affects the coefficients (24), (25) and the entanglement evolution. According to the definition [29, 30], the negativity is calculated as

$$
E_{A 1 A 2}(t)=\sum_{\mu=1}^{4} \max \left(0,-2 x_{\mu}\right),
$$

where $x_{\mu}(\mu=1,2,3,4)$ are the four roots of Eq. (22). 
For $n=0$ and $\Delta=0$, the negativity versus the scaled time $T=\lambda_{2} t$ is plotted in Fig. 1. The solid and dashed lines correspond to $\varphi=\pi / 2$ and $\varphi=0$ (or $\varphi=\pi$ ), respectively. For other values of $0<\varphi<\pi / 2$ or $\pi / 2<\varphi<\pi$, the negativity lies between these two curves. The negativity changes periodically with the period $\pi$ for the scaled time. The figure is plotted within one period. The phase enhances the entanglement obviously. Around the maximum entanglement, the quantum phase makes the entanglement evolution slower, which means the state becomes more stable or robust against external effects. Near zero entanglement, the phase makes the entanglement change faster.

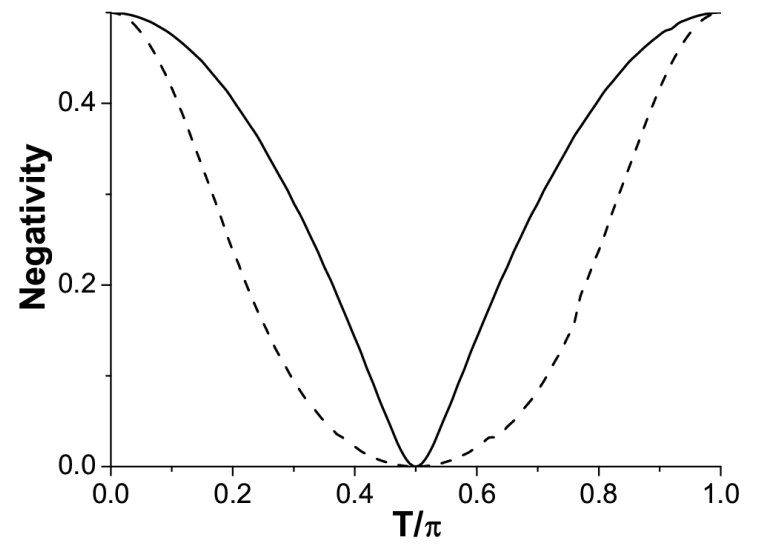

Fig. 1. Negativity for atoms $A 1$ and $A 2$ versus the scaled time $T$ over $\pi$ for $n=0, \Delta=0$ and $\varphi=0$ (dashed line), $\varphi=\pi / 2$ (solid line).

In Fig. 1, the negativity $E_{A 1 A 2}(T)$ is zero at $T=\pi / 2$. Calculations show that the initial atom-atom entanglement is transferred to atom $A 1$ and the field. At this time, the wave function (19) becomes

$$
\begin{aligned}
& \left|\psi_{A 1 A 2 F}(t)\right\rangle=\left|g_{2}\right\rangle\left[\sqrt{q}\left(\cos \alpha\left|g_{1}\right\rangle|0\rangle+\sin \alpha\left|e_{1}\right\rangle|1\rangle\right)\right. \\
& \left.-\mathrm{i} \sqrt{1-q} \mathrm{e}^{\mathrm{i} \theta}\left|g_{1}\right\rangle|1\rangle\right] .
\end{aligned}
$$

Atom $A 2$ is disentangled from the subsystem formed by atom $A 1$ and the field. The entanglement between atom $A 1$ and the field is equal to the initial atom-atom entanglement. Or, the initial atom-atom entanglement is completely transferred to the atom-field system.

For $n=0$, there is no ESD. For $n=1$, ESD happens as shown in Fig. 2. It is seen that the quantum phase delays ESD, hastens the entanglement revival, and shortens the death time or the time of zero entanglement. Between $T=1.5 \pi$ and $T>2 \pi$, the quantum phase even induces a small peak for the negativity. After ESD, the maximum entanglement can reach 0.45 for $\varphi=0$ and 0.47 for $\varphi=+\pi / 2$.

Next we consider a case that the detuning and the quantum phase exist together. The parameters are chosen as $q=0.7, n=1$ and $\alpha=1.2 \pi / 4$. The numerical results are shown in Fig. 3. The short dashed, long dashed and solid lines correspond to (a) $\Delta=0, \varphi=0$,

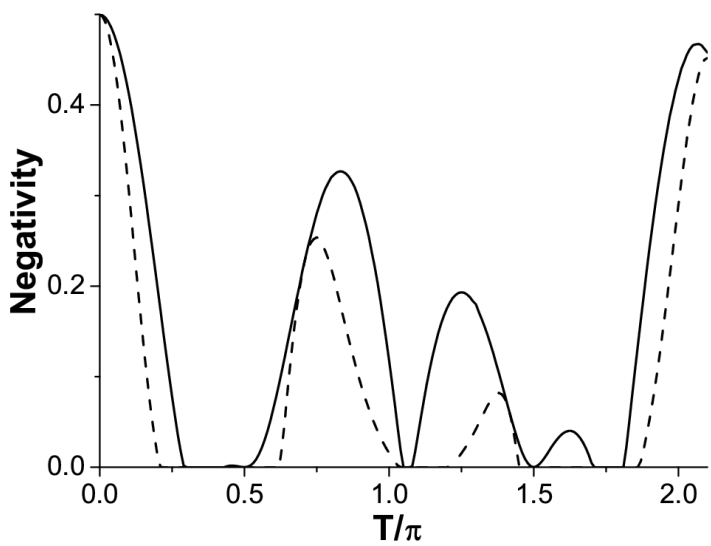

Fig. 2. Negativity for atoms $A 1$ and $A 2$ versus the scaled time $T$ over $\pi$ for $n=1, \Delta=0$ and $\varphi=0$ (dashed line), $\varphi=\pi / 2$ (solid line).

(b) $\Delta=0.4 \lambda_{2}, \varphi=0$ and (c) $\Delta=0.4 \lambda_{2}, \varphi=\pi / 2$, respectively. One sees that detuning slightly enhances the entanglement. The quantum phase modifies the entanglement more greatly, especially when the negativity is not near zero. One sees that for different parameters, the quantum phase changes the behavior of the entanglement evolution obviously.

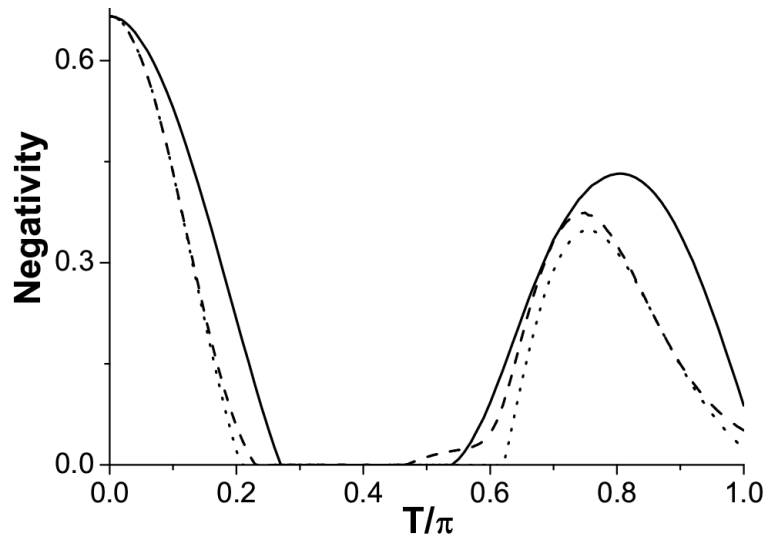

Fig. 3. Negativity for atoms $A 1$ and $A 2$ versus the scaled time $T$ over $\pi$ for $q=0.7, n=1, \alpha=1.2 \pi / 4$ and (a) $\Delta=0, \varphi=0$ (dotted line), (b) $\Delta=0.4 \lambda_{2}$, $\varphi=0$ (dashed line), (c) $\Delta=0.4 \lambda_{2}, \varphi=\pi / 2$ (solid line).

Now we turn to the atom-field entanglement. Tracing over the atomic states of $A 2$, we get the reduced density matrix $\rho_{A 1 F}(t)$ for $A 1$ and the field from $\rho_{A 1 A 2 F}(t)=$ $\left|\psi_{A 1 A 2 F}(t)\right\rangle\left\langle\psi_{A 1 A 2 F}(t)\right|$. Atom $A 1$ is a qubit with the states $\left(\left|e_{1}\right\rangle,\left|g_{1}\right\rangle\right)$ and the field is a qutrit with the states $(|n+1\rangle,|n\rangle,|n-1\rangle)$. Atom $A 1$ and the field form a qubit-qutrit system. The entanglement can still be measured by the negativity $[18,19]$. In the basis $\left(\left|e_{1}\right\rangle|n+1\rangle\right.$, $\left.\left|e_{1}\right\rangle|n\rangle,\left|e_{1}\right\rangle|n-1\rangle,\left|g_{1}\right\rangle|n+1\rangle,\left|g_{2}\right\rangle|n\rangle,\left|g_{1}\right\rangle|n-1\rangle\right)$, the matrix form for $\rho_{A 1 F}(t)$ is 


$$
\rho_{A 1 F}(t)=\left[\begin{array}{cccccc}
\rho_{11} & 0 & 0 & \rho_{14} & \rho_{15} & 0 \\
0 & \rho_{22} & 0 & 0 & \rho_{25} & \rho_{26} \\
0 & 0 & 0 & 0 & 0 & 0 \\
\rho_{41} & 0 & 0 & \rho_{44} & \rho_{45} & 0 \\
\rho_{51} & \rho_{52} & 0 & \rho_{54} & \rho_{55} & \rho_{56} \\
0 & \rho_{62} & 0 & 0 & \rho_{65} & \rho_{66}
\end{array}\right],
$$

where the nonzero matrix elements are

$$
\begin{aligned}
& \rho_{11}=q\left|a_{e g}(n, t) \sin \alpha\right|^{2}, \quad \rho_{22}=q\left|a_{e e}(n, t) \sin \alpha\right|^{2}, \\
& \rho_{44}=(1-q)\left|a_{e g}(n, t)\right|^{2}, \quad \rho_{66}=q\left|a_{g e}(n, t) \cos \alpha\right|^{2}, \\
& \rho_{55}=q\left|a_{g g}(n, t) \cos \alpha\right|^{2}+(1-q)\left|a_{e e}(n, t)\right|^{2}, \\
& \rho_{14}=\rho_{41}^{*}=\sqrt{q(1-q)}\left|a_{e g}(n, t)\right|^{2} \mathrm{e}^{\mathrm{i}(\varphi-\theta)} \sin \alpha, \\
& \rho_{15}=\rho_{51}^{*}=q a_{e g}(n, t) a_{g g}^{*}(n, t) \cos \alpha \sin \alpha, \\
& \rho_{45}=\rho_{54}^{*}=\sqrt{q(1-q)} a_{e g}(n, t) a_{g g}^{*}(n, t) \mathrm{e}^{\mathrm{i} \theta}, \\
& \rho_{25}=\rho_{52}^{*}=\sqrt{q(1-q)}\left|a_{e e}(n, t)\right|^{2} \mathrm{e}^{\mathrm{i}(\varphi-\theta)} \sin \alpha, \\
& \rho_{26}=\rho_{62}^{*}=q a_{e e}(n, t) a_{g e}^{*}(n, t) \mathrm{e}^{\mathrm{i} \varphi} \cos \alpha \sin \alpha, \\
& \rho_{56}=\rho_{65}^{*}=\sqrt{q(1-q)} a_{e e}(n, t) a_{g e}^{*}(n, t) \mathrm{e}^{\mathrm{i} \theta} \cos \alpha . \quad(29)
\end{aligned}
$$

For $q=1$, the density matrix (27) is greatly simplified, which reduces to two independent $X$-type matrices formed by the matrix elements $\left(\rho_{11}, \rho_{55}, \rho_{15}, \rho_{51}\right)$ and $\left(\rho_{22}, \rho_{66}, \rho_{26}, \rho_{62}\right)$. In this case the phases have no effects on the entanglement. Generally, the partial transpose of the reduced density matrix (27) is

$$
\rho_{A 1 F}^{(\mathrm{PT})}(t)=\left[\begin{array}{cccccc}
\rho_{11} & 0 & 0 & \rho_{41} & 0 & 0 \\
0 & \rho_{22} & 0 & \rho_{51} & \rho_{52} & 0 \\
0 & 0 & 0 & 0 & \rho_{62} & 0 \\
\rho_{14} & \rho_{15} & 0 & \rho_{44} & \rho_{45} & 0 \\
0 & \rho_{25} & \rho_{26} & \rho_{54} & \rho_{55} & \rho_{56} \\
0 & 0 & 0 & 0 & \rho_{65} & \rho_{66}
\end{array}\right] .
$$

For each set of matrix elements, it has six eigenvalues which can be calculated numerically. The negativity is derived similarly to (26). When $n=0$ and $\Delta=0$, the negativity for $A 1$-field entanglement versus the scaled time is plot in Fig. 4. The phase makes the entanglement grow faster around $T=0$ and degrade more quickly around $T=\pi$. The time interval for the negativity larger than half the maximum value 0.5 is $\Delta T=0.38 \pi$ when the phase is zero and $\Delta T=0.52 \pi$ when the phase is $\pi / 2$. The phase makes the system stay a longer time in the stronger entanglement state. The increased time by percent is about $(0.52 \pi-0.38 \pi) /(0.38 \pi) \approx 37 \%$. In case of detuning or $\Delta \neq 0$, the maximum negativity cannot reach 0.5 , which means that the entanglement transfer is incomplete when there is detuning. The maximum values of the negativity are 0.494 and 0.474 when $\Delta=0.2 \lambda_{2}$ and $0.4 \lambda_{2}$ for $\varphi=0$. The maximum values of the negativity increases to 0.496 and 0.488 when $\varphi=\pi / 2$.

Calculations show that the A2-field entanglement is not affected by the quantum phases. In the above discussions, atom $A 2$ interacts with the external field. If atom $A 1$ interacts with the external field or the two atoms interact with two separate cavity fields, similar results are obtained: the phase $\varphi$ affects the atom-atom and atomfield entanglement, and the phase $\theta$ does not.

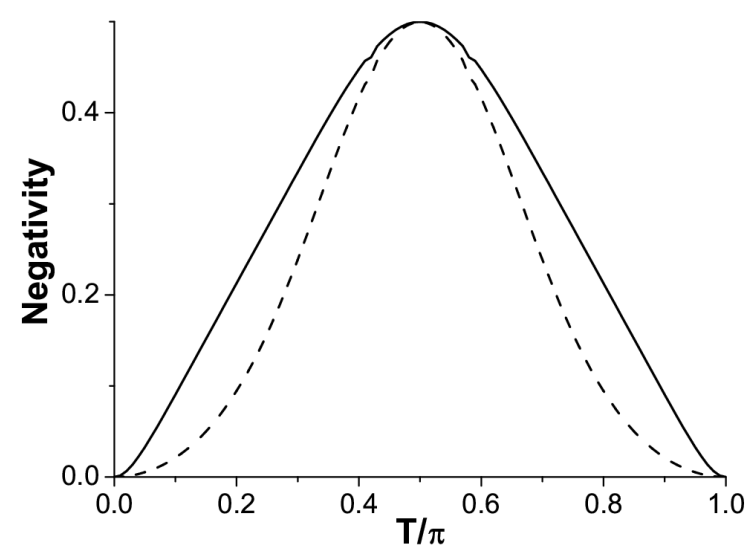

Fig. 4. Negativity for atom $A 1$ and the field versus the scaled time $T$ over $\pi$ for $n=0, \Delta=0$, and $\varphi=0$ (dashed line), $\varphi=\pi / 2$ (solid line).

\section{Summary}

Time evolution of entanglement in systems of two atoms and one cavity field are investigated. The quantum phase can enhance the atom-atom and atom-field entanglement. ESD can be delayed and the time of zero entanglement can be shortened. The time that the system stays in stronger entanglement states is extended. Whether the quantum phase really affects the entanglement evolution depends on the concrete forms of wave functions or density matrices.

\section{References}

[1] D. Bouwmeester, J.W. Pan, H. Weinfurter, A. Zeilinger, J. Mod. Opt. 47, 279 (2000).

[2] M.A. Nielsen, I.L. Chuang, Quantum Computation and Quantum Information, Cambridge University Press, Cambridge 2000.

[3] A. Galindo, M.A. Martin-Delgado, Rev. Mod. Phys. 74, 347 (2002).

[4] R. Horodecki, P. Horodecki, M. Horodecki, K. Horodecki, Rev. Mod. Phys. 81, 865 (2009).

[5] K. Życzkowski, P. Horodecki, M. Horodecki, R. Horodecki, Phys. Rev. A 65, 012101 (2001).

[6] T. Yu, J.H. Eberly, Phys. Rev. Lett. 93, 140404 (2004).

[7] T. Yu, J.H. Eberly, Phys. Rev. Lett. 97, 140403 (2006).

[8] F. Benatti, R. Floreanini, M. Piani, Phys. Rev. Lett 91, 070402 (2003).

[9] F. Benatti, R. Floreanini, J. Phys. A, Math. Gen. 39, 2689 (2006).

[10] A. Kowalewska-Kudłaszyk, W. Leoński, J. Phys. B, At. Mol. Opt. Phys. 43, 205503 (2010).

[11] M. Bartkowiak, A. Miranowicz, X.G. Wang, Y.X. Liu, W. Leonski, F. Nori, Phys. Rev. A 83, 053814 (2011).

[12] A. Kowalewska-Kudłaszyk, W. Leoński, J. Peina, Phys. Rev. A 83, 052326 (2011). 
[13] A. Kowalewska-Kudłaszyk, W. Leoński, Phys. Scr. T 140, 014051 (2010).

[14] Z. Ficek, R. Tanas, Phys. Rep. 372, 369 (2002).

[15] J.I. Cirac, P. Zoller, Phys. Rev. A 50, 2799R (1994).

[16] E.T. Jaynes, F.W. Cummings, Proc. IEEE 51, 89 (1963).

[17] T. Yu, J.H. Eberly, Opt. Commun. 264, 393 (2006).

[18] M. Yäonac, T. Yu, J.H. Eberly, J. Phys. B 39, S621 (2006).

[19] Z.J. Li, J.Q. Li, Y.H. Jin, Y.H. Nie, J. Phys. B 40, 3401 (2007).

[20] Z.X. Man, Y.J. Xia, N.B. An, J. Phys. B 41, 085503 (2008).

[21] S. Chan, M.D. Reid, Z. Ficek, J. Phys. B 42, 065507 (2009).

[22] W.B. Cardoso, A.V. Avelar, B. Baseia, N.G. de Almeida, J. Phys. B 42, 195507 (2009).

[23] J. Leon, C. Sabin, Phys. Rev. A 79, 012301 (2009).

[24] Z. Ficek, Front. Phys. China 5, 26 (2010).
[25] P.J. dos Reis, S.S. Sharma, N.K. Sharma, arXiv:quant-ph/1106.2570v2 (2011).

[26] K. Hunkar, Commun. Theor. Phys. 56, 487 (2011).

[27] Q.L. He, J.B. Xu, Opt. Commun. 284, 1714 (2011).

[28] M.P. Almeida, F. de Melo, M. Hor-Metll, A. Salles, S.P. Walborn, P.H.S. Ribeiro, L. Davidovich, Science 316, 597 (2007).

[29] A. Peres, Phys. Rev. Lett. 77, 1413 (1996).

[30] M. Horodecki, P. Horodecki, R. Horodecki, Phys. Lett. A 223, 1 (1996).

[31] H. Hill, W.K. Wootters, Phys. Rev. Lett. 78, 5022 (1997).

[32] W.K. Wootters, Phys. Rev. Lett. 80, 2245 (1998).

[33] K.M. O'Connor, W.K. Wootters, Phys. Rev. A 63, 052302 (2001).

[34] C.C. Gerry, Phys. Rev. A 53, 4591 (1996).

[35] C.C. Gerry, Phys. Rev. A 53, 2857 (1996). 